Pacific Journal of Mathematics

BANACH SPACES WHICH SATISFY LINEAR IDENTITIES 


\section{BANACH SPACES WHICH SATISFY LINEAR IDENTITIES}

\section{BRUCE REZNICK}

In 1935, Jordan and von Neumann proved that any Banach space which satisfies the parallelogram law

$$
\begin{aligned}
\|x+y\|^{2}+\|x-y\|^{2}= & 2\left(\|x\|^{2}+\|y\|^{2}\right) \\
& \text { for all elements } x \text { and } y
\end{aligned}
$$

must be a Hilbert space.

Subsequent authors have found norm conditions weaker than (1) which require a Banach space to be a Hilbert space. Notable examples include the results of Day, Lorch, Senechalle and Carlsson.

In this paper, we study nontrivial linear identities such as

(2) $\sum_{k=0}^{m} a_{k}\left\|c_{k}(0) x_{0}+\cdots+c_{k}(n) x_{n}\right\|^{p}=0$ for all elements $x_{i}$

on a Banach space $X$.

A necessary condition for (2) to hold in $X$ is that $\|x+t y\|^{p}$ must be a polynomial in $t$ for all choices of elements $x$ and $y$. A sufficient condition for (2) to hold in $X$ is that (2) must hold in the field of scalars. Specific identities are presented including a generalized parallelopiped law first observed by Koehler, and some isometric results are stated.

2. The parallelogram law revisited. In 1909 [4], Fréchet proved the following result.

LEMMA 1 (Fréchet). If $g$ is continuous function on $\boldsymbol{R}$ and, for all real $r$ and $s$, equation (3) holds, then $g$ is a polynomial with degree less than $N$.

$$
\sum_{k=0}^{N}(-1)^{N-k}\left(\begin{array}{c}
N \\
k
\end{array}\right) g(r+k s)=0
$$

Proof. It is well-known that any sequence $\left\{a_{n}\right\}$ satisfying $\Sigma(-1)^{N-k}\left(\begin{array}{l}N \\ k\end{array}\right) a_{k+M}=0$ for all $M$ is generated by a polynomial; that is, there is a polynomial $P$ with degree less than $N$ for which $a_{n}=$ $P(n)$.

In (3), put $g(n)=a_{n}, s=1$ and let $r$ range over the integers. Then there is a polynomial $P$ with $P(n)=a_{n}=g(n)$. Now put $g(n / 2)=b_{n}, s=1 / 2$ and let $r$ range over the half-integers. There is 
a polynomial $Q$ with $Q(n)=b_{n}=g(n / 2)$. Thus $Q(2 n)=P(n)$ for all $n$ and $Q(x) \equiv P(x / 2)$, so $g(n / 2)=P(n / 2)$. A repetition of this argument demonstrates that $P\left(n 2^{-m}\right)=g\left(n 2^{-m}\right)$ for all integers $m$ and $n$. By the continuity of $g, P(x)=g(x)$ for all $x$ and the lemma is proved.

The parallelogram law has a second difference nature: $\|x+y\|^{2}-2\|x\|^{2}+\|x-y\|^{2}=2\|y\|^{2}$, (see Johnson [6]). Putting successively $x=u+2 v, y=v$ and $x=u+v, y=v$ and subtracting, we get (4). Fix $w$ and $z$, elements of any space in which (1) holds.

$$
\sum_{k=0}^{3}(-1)^{k}\left(\begin{array}{l}
3 \\
k
\end{array}\right)\|u+k v\|^{2}=0 \text {. }
$$

Let $u=w+r z, v=s z$ and substitute in (4). Setting $\|w+t z\|^{2}=$ $g(t)$, we obtain (5). By the triangle inequality, $\left|g^{1 / 2}\left(t_{0}\right)-g^{1 / 2}\left(t_{1}\right)\right| \leqq$ $\left|t_{1}-t_{0}\right| \cdot\|z\|$, hence $g$ is continuous.

$$
\sum_{k=0}^{3}(-1)^{k}\left(\begin{array}{l}
3 \\
k
\end{array}\right) g(r+k s)=0 .
$$

Applying Lemma 1 to (5) we see that $g(t)$ is quadratic in $t$. Indeed, if $\|w+t z\|^{2}=A(w, z)+2 B(w, z) t+C(w, z) t^{2}$, then clearly $A(w, z)=$ $\|w\|^{2}$ and $C(w, z)=\|z\|^{2}$. It is not hard to verify that $B(w, z)$ satisfies the definition of a real inner-product and $B(w, z)+i B(w, i z)$ that of a complex inner-product. This provides an alternative proof to the Jordan-von Neumann theorem.

We shall return to the parallelogram law in $\S 6$ as an embarkation point for a series of linear identities which hold in more spaces than Hilbert space. As an appetizer, consider (6), a generalization of (1) to a three-dimensional parallelopiped.

$$
\begin{aligned}
\|x+y+z\|^{k} & +\|x+y-z\|^{k}+\|x-y+z\|^{k}+\|x-y-z\|^{k} \\
& -2\left(\|x+y\|^{k}+\|x-y\|^{k}+\|x+z\|^{k}+\|x-z\|^{k}\right. \\
& \left.+\|y+z\|^{k}+\|y-z\|^{k}\right) \\
& +4\left(\|x\|^{k}+\|y\|^{k}+\|z\|^{k}\right)=0 .
\end{aligned}
$$

Observe that (6) holds for $k=2$ in Hilbert space and for $k=4$ in Hilbert space and in $L_{4}(x, \mu)$ for any $(x, \mu)$. Indeed, it may be verified that (6) holds in any Banach space in which $\|r x+s y+t z\|^{4}$ is a homogeneous polynomial in $r, s$, and $t$ for fixed elements $x, y$, and $z$. This condition turns out to be necessary as well, and the situation will prove to be typical.

3. Spaces which satisfy linear identities. The main result of this section will be Theorem 4, which follows from Theorem 2 by an intervening lemma. 
THEOREM 2. Suppose $X$ is a Banach space in which (7) holds for all elements $x$ and $y$, where $a_{k} \neq 0, p>0, b_{0}=0$ and the $b_{k}$ 's are distinct.

$$
\sum_{k=0}^{m} a_{k}\left\|x+b_{k} y\right\|^{p}=0 .
$$

Then for every $x$ and $y$ in $X,\|x+t y\|^{p}$ is a polynomial in $t$. In particular, $p$ is an even integer.

Proof. Set $A(u, v)=a_{0}\|u\|^{p}+\sum_{k=1}^{m}\left\|u+b_{k} v\right\|^{p}$. By the hypotheses, $A(u, v)=0$ for all elements $u$ and $v$ and $b_{k} \neq 0$ for $k \geqq 1$. Fix elements $x$ and $y$; let $z$ be arbitrary. Then $0=A\left(x+z, y-b_{1}^{-1} z\right)-$ $A(x, y)$. Writing this out, we obtain (8). Notice that the term for $k=1$ in the sum in (8) vanishes.

$$
\begin{aligned}
0= & a_{0}\left(\|x+z\|^{p}-\|x\|^{p}\right) \\
& +\sum_{k=1}^{m} a_{k}\left(\left\|x+b_{k} y+\left(1-b_{k} b_{1}^{-1}\right) z\right\|^{p}-\left\|x+b_{k} y\right\|^{p}\right) .
\end{aligned}
$$

We repeat this procedure (due to Wilson [14]) and obtain (9), where the inner sum is taken over all choices of $1 \leqq i_{1}<\cdots<i_{j} \leqq m$.

$$
0=\sum_{j=0}^{m}(-1)^{j}\left(\Sigma A\left(x+j z, y-\left(b_{i_{1}}^{-1}+\cdots+b_{i_{j}}^{-1}\right) z\right)\right) .
$$

Equation (9) may be expanded using the definition of $A(u, v)$ as (10), where the inner sum is as before.

$$
\begin{aligned}
0= & \sum_{j=0}^{m}(-1)^{j}\left(\begin{array}{c}
m \\
j
\end{array}\right)\|x+j z\|^{p} \\
& +\sum_{k=1}^{m} a_{k} \sum_{j=0}^{m}(-1)^{j}\left(\Sigma\left\|x+b_{k} y+\left(j-b_{k}\left(b_{i_{1}}^{-1}+\cdots+b_{i_{j}}^{-1}\right)\right) z\right\|^{p}\right) .
\end{aligned}
$$

For any fixed $k$, the $2^{m}$ subsets of $\{1, \cdots, m\}$ divide into two corresponding classes: if $k \notin I=\left\{i_{1}, \cdots, i_{j}\right\}$, then $I$ and $I \cup\{k\}$ are associated. Using this pairing, the terms

$$
(-1)^{j}\left\|x+b_{k} y+\left(j-b_{k}\left(b_{i_{1}}^{-1}+\cdots+b_{i_{j}}^{-1}\right)\right) z\right\|^{p}
$$

and $(-1)^{j+1}\left\|x+b_{k} y+\left(j+1-b_{k}\left(b_{i_{1}}^{-1}+\cdots+b_{i_{j}}^{-1}+b_{k}^{-1}\right)\right) z\right\|^{p}$ cancel out in the triple sum. Hence, (10) reduces to (11).

$$
0=\sum_{j=0}^{m}(-1)^{j}\left(\begin{array}{c}
m \\
j
\end{array}\right)\|x+j z\|^{p} .
$$

As in $\S 2$, we choose $u$ and $v$ arbitrary nonzero elements in $X$, let $r$ and $s$ be arbitrary reals and set $x=u+r v, y=s v$ and $g(t)=$ 
$\|u+t v\|^{p}$ in (11). We obtain (12), and by Lemma $1, g(t)=\|u+t v\|^{p}$ must be a polynomial.

$$
0=\sum_{j=0}^{m}(-1)^{j}\left(\begin{array}{c}
m \\
j
\end{array}\right) g(r+j s) .
$$

As $t^{-p} g(t) \rightarrow\|v\|^{p} \neq 0, g$ has degree $p$ and as $g(t) \geqq 0, p$ is an even integer.

Lemma 3. Suppose $X$ is a Banach space in which (13) holds for all elements $x$ and $y$, where $\alpha_{k} \neq 0, p>0$ and the $\left(b_{k}, c_{k}\right)$ 's are pairwise linearly independent.

$$
\sum_{k=0}^{m} a_{k}\left\|b_{k} x+c_{k} y\right\|^{p}=0 .
$$

Then $\|x+t y\|^{p}$ is a polynomial in $t$ for all elements $x$ and $y$.

Proof. We shall reduce (13) to (7). Permute the $k$ 's so that $\left|b_{0}\right| \leqq \cdots \leqq\left|b_{m}\right|$ and let $\mathrm{d}_{k}=b_{k}^{-1} c_{k}$. If $b_{0} \neq 0$ then $b_{k} \neq 0$; if $b_{0}=0$ then $c_{0} \neq 0$ and $b_{k} \neq 0$ for $k \geqq 1$ and the $d_{k}$ 's are distinct by the linear independence. In the first case, rewrite (13) as (14) and then put into it $x=u-d_{0} v, y=v$ where $u$ and $v$ are arbitrary.

$$
\sum_{k=0}^{m} a_{k}\left|b_{k}\right|^{p}\left\|x+d_{k} y\right\|^{p}=0 \text {. }
$$

We obtain (15) which is in the form of (7).

$$
\sum_{k=0}^{m} a_{k}\left|b_{k}\right|^{p}\left\|u+\left(d_{k}-d_{0}\right) v\right\|^{p}=0 .
$$

In the second case, let $s$ be a number for which $b_{k}+s c_{k} \neq 0$ and put $x=u, y=s u+v$ into (13) where $u$ and $v$ are again arbitrary. (If $\left(b_{j}, c_{j}\right)$ and $\left(b_{k}, c_{k}\right)$ are independent then so are $\left(b_{j}+s c_{j}, c_{j}\right)$ and $\left(b_{k}+s c_{k}, c_{k}\right)$.) We obtain (16) which now falls under the first case.

$$
\sum_{k=0}^{m} a_{k c}\left\|\left(b_{k}+s c_{k}\right) u+c_{k} v\right\|^{p}=0 .
$$

Carlsson [1] proved Lemma 3 for $p=2$ and $a_{k}, b_{k}, c_{k}$ real.

THEOREM 4. Suppose $X$ is a Banach space in which (17) holds for all elements $x_{i}$, where $a_{k} \neq 0, p>0$, the $\left(c_{k}(0), \cdots, c_{k}(n)\right)$ 's are pairwise linearly independent $(n+1)$-tuples, and for every $i$ there is at least one $k$ with $c_{k}(i) \neq 0$.

$$
\sum_{k=0}^{m} a_{k}\left\|c_{k}(0) x_{0}+\cdots+c_{k}(n) x_{n}\right\|^{p}=0 .
$$


Then, for all $x$ and $y,\|x+t y\|^{p}$ is a polynomial in $t$.

Proof. Permute the $k$ 's so that $\left|c_{0}(0)\right| \geqq\left|c_{1}(0)\right| \geqq \cdots \geqq\left|c_{m}(0)\right|$, then $c_{0}(0) \neq 0$. If $c_{k}(0)=0$ for $k \geqq 1$, put $x_{1}=\cdots=x_{n}=0$ in (17), then $a_{0}\left\|c_{0}(0) x_{0}\right\|^{p}=0$ for all $x_{0}$, that is, $X$ is trivial. Otherwise, suppose $c_{k}(0) \neq 0$ for $k \leqq j, j \geqq 1$ and (17) may be rewritten as (18), where $d_{k}(i)=c_{k}(i) c_{k}(0)^{-1}$.

$$
\begin{gathered}
\sum_{k=0}^{j} a_{k}\left|c_{k}(0)\right|^{p}\left\|x_{0}+d_{k}(1) x_{1}+\cdots+d_{k}(n) x_{n}\right\|^{p} \\
+\sum_{k=j+1}^{m} a_{k}\left\|c_{k}(1) x_{1}+\cdots+c_{k}(n) x_{n}\right\|^{p}=0 .
\end{gathered}
$$

From the linear independence, it follows that the $n$-tuples $\left\{\left(d_{k}(1)\right.\right.$, $\left.\left.\cdots, d_{k}(n)\right)\right\}$ are distinct. Define the sets

$$
A_{i k}=\left\{\left(s_{1}, \cdots, s_{n}\right) \in C^{n} \mid \sum_{q=1}^{n} s_{q} d_{i}(q)=\sum_{q=1}^{n} s_{q} d_{k}(q)\right\} .
$$

These sets do not exhaust $C_{n}^{n}$ and so we can find $r_{q}$ for which the $b_{k}$, defined by $b_{k}=\sum_{q=1}^{n} r_{q} d_{k}(q)$, are distinct.

For arbitrary $x$ and $y$, let $x_{0}=x, x_{k}=r_{k} y$ in (18). We obtain (19) which is in the form of (13).

$$
\sum_{k=0}^{j} \alpha_{k}\left|c_{k}(0)\right|^{p}\left\|x+b_{k} y\right\|^{p}+\left(\sum_{k=\rho+1}^{m} \sum_{i=1}^{n}\left|c_{k}(i) r_{i}\right|^{p}\right)\|y\|^{p}=0 .
$$

This completes the proof.

To obtain the natural corollary we need a lemma.

LEMma 5. Suppose $f$ is a nonnegative function and $q$ and $r$ are positive integers such that $f^{a}$ and $f^{r}$ are polynomials. Then $f^{s}$ is a polynomial, where $s=(q, r)$, the greatest common divisor.

Proof. We have $f^{q}(x)=A^{q} \prod_{i=1}^{k}\left(x-x_{i}\right)^{\lambda_{i}}, x_{i} \neq x_{j}, A \geqq 0$. Thus, $f^{r}(x)=A^{r} \prod_{i=1}^{k}\left(x-x_{i}\right)^{\lambda_{i} r / q}$, and so $q \mid r \lambda_{i}$. Write $q=s \bar{q}, r=s \bar{r},(\bar{q}, \bar{r})=$ 1. As $s \bar{q}\left|s \bar{r} \lambda_{i}, \bar{q}\right| \lambda_{i}$ and so $f^{s}=A^{s} \prod_{i=1}^{k}\left(x-x_{i}\right)^{\lambda_{i} / \bar{q}}$ is a polynomial.

Corollary 6. Suppose $X$ is a Banach space which satisfies (20) for all elements $x_{i}$, where $p_{k}>0$ and the same restrictions on constants apply as in Theorem 4.

$$
\sum_{k=0}^{m} a_{k}\left\|c_{k}(0) x_{0}+\cdots+c_{k}(n) x_{n}\right\|^{p_{k}}=0 .
$$

Then $p_{k}$ is an even integer for each $k$ and $\|x+t y\|^{p}$ is a polynomial 
in $t$ for all $x$ and $y$, where $p=\left(p_{1}, \cdots, p_{k}\right)$, the greatest common divisor.

Proof. Fix $y_{0}, \cdots, y_{n}$ and let $b_{k}=a_{k}\left\|c_{k}(0) y_{0}+\cdots+c_{k}(n) y_{n}\right\|^{p_{k}}$. Let $x_{k}=\lambda y_{k}$ for $\lambda>0$, then by (20), $\sum_{k=0}^{m} b_{k} \lambda^{p_{k}}=0$. Upon collecting

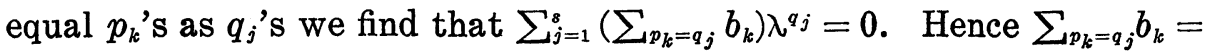
0 . Thus $X$ satisfies an equation of form (17) for each $p_{k}$ and $\|x+t y\|^{p_{k}}$ is a polynomial in $t$. We now apply Lemma 5 to this situation and conclude that $f^{p}$ is a polynomial.

4. The Class $\mathscr{P}_{2 n}$, The necessary condition of Theorem 4 suggests the following definition: a Banach space $X$ is polynomial of degree $2 n$ if, for all elements $x$ and $y,\|x+t y\|^{2 n}$ is a polynomial of degree $2 n$ in real $t$. The class $\mathscr{P}_{2 n}$ consists of all Banach spaces which are polynomial of degree $2 n$.

Theorem 7. (i) If $X$ is in $\mathscr{P}_{2}$, then $X$ is a Hilbert space.

(ii) If $m$ divides $n$, then $\mathscr{P}_{2 m}$ is contained in $\mathscr{P}_{2 n}$.

(iii) If $\boldsymbol{r}=(m, n)$, then $\mathscr{P}_{2 m} \cap \mathscr{P}_{2 n}=\mathscr{P}_{2 r}$.

(iv) If $k$ is an integer dividing $n$, then for all measure spaces $(X, \mu), L_{2 k}(X, \mu)$ is in $\mathscr{P}_{2 n}$.

( $v)$ If $p$ is not an even integer and $(X, \mu)$ is not trivial, then $L_{p}(X, \mu)$ is not in $\mathscr{P}_{2 n}$ for any $n$.

Proof. (i) This is the Jordan-von Neumann theorem; see also $\S 2$.

(ii) If $\|x+t y\|^{2 m}$ is a polynomial and $2 n / 2 m$ is an integer, then $\|x+t y\|^{2 n}$ is a polynomial.

(iii) Combine (ii) and Lemma 5.

(iv) It suffices to show that $L_{2 n}(X, \mu)$ is in $\mathscr{P}_{2 n}$. Pick elements $f$ and $g$ in $L_{2 n}(X, \mu)$; then

$$
\begin{aligned}
\|f+t g\|^{2 n} & =\int|f+t g|^{2 n} d \mu=\int\left(|f|^{2}+t(f \bar{g}+\bar{f} g)+t^{2}\right)^{n} d \mu \\
& =\int \Sigma c_{i}(f, g) t^{i} d \mu,
\end{aligned}
$$

where $c_{i}(f, g)$ is a sum of terms of the form $f^{a} \bar{f}^{b} g^{c} \bar{g}^{d}$ with $c+d=i$, $a+b=2 n-i$. As $\int|f|^{2 n} d \mu<\infty$ and $\int|g|^{2 n} d \mu<\infty$, each $c_{i}(f, g)$ is integrable by Hölder's inequality. Thus $\|f+t g\|^{2 n}=\Sigma \int c_{i}(f, g) d \mu \cdot t^{i}$ and $L_{2 n}(X, \mu)$ is in $\mathscr{P}_{2 n}$.

(v) If $(X, \mu)$ has two disjoint sets of positive measure, then one may easily construct elements $x$ and $y$ in $L_{p}(X, \mu)$ with $\|x+t y\|^{p}=$ $1+|t|^{p}$. If $p$ is not an even integer then $\left(1+|t|^{p}\right)^{2 n / p}$ is not a 
polynomial as it is not in $C^{[p]+1}$. (See also Sundaresan [13].) The embedding properties of $\mathscr{P}_{2 n}$ will be described in a subsequent paper [11]. We state without proof the following theorem.

THEOREM 8. (i) If $X$ is a real two-dimensional space in $\mathscr{F}_{4}$, then it is isometrically isomorphic to a subspace of $L_{4}[0,1]$.

(ii) There exists a three-dimensional space in $\mathscr{P}_{4}$ which is not isometrically isomorphic to any subspace of $L_{4}(X, \mu)$ for any $(X, \mu)$.

(iii) There exist two-dimensional spaces in $\mathscr{P}_{2 n}(n \geqq 3)$ which are not isometrically isomorphic to any subspace of $L_{2 n}(X, \mu)$ for any $(X, \mu)$.

5. The sufficient condition. In this section, we prove that the classes $\mathscr{P}_{2 n}$ form the finest possible gradation of Banach spaces according to the linear identities they satisfy: if an identity of type (16) holds with $p=2 n$ for one space in $\mathscr{P}_{2 n}$ then it holds for all spaces in $\mathscr{P}_{2 n}$. We begin with a few preliminaries.

Lemma 9. Suppose a function $g\left(u_{1}, \cdots, u_{n}\right)$ is a polynomial in each of its variables separately, that is, (21) holds for each $r$, $1 \leqq r \leqq n$, where the $g_{k, r}$ 's are continuous and a carat over $a$ variable signifies its omission.

$$
g\left(u_{1}, \cdots, u_{n}\right)=\sum_{k=1}^{s_{r}} g_{k, r}\left(u_{1}, \cdots, \hat{u}_{r}, \cdots, u_{n}\right) u_{r}^{k} .
$$

Then $g$ is in fact a polynomial in the variables together.

Proof. The proof will be by induction on $n$. The theorem is certainly true for $n=1$. Suppose it is true if $n=m$. For $n=$ $m+1$ we have by hypothesis a representation of $g\left(u_{1}, \cdots, u_{m+1}\right)$ in the form (22). Let $M=\max s_{r}+1$.

$$
g\left(u_{1}, \cdots, u_{m+1}\right)=\sum_{k=1}^{m-1} g_{k, m+1}\left(u_{1}, \cdots, u_{m}\right) u_{m+1}^{k} .
$$

Define the $M$ th difference $\Delta^{M} h(r, v)\left(u_{1}, \cdots, u_{s}\right)$ by $(23)$.

$$
\Delta^{M} h(r, v)\left(u_{1}, \cdots, u_{s}\right)=\sum_{i=0}^{M}(-1)^{M-i}\left(\begin{array}{c}
M \\
i
\end{array}\right) h\left(u_{1}, \cdots, u_{r}+i v, \cdots, u_{s}\right) .
$$

Since $\Delta^{M}$ is certainly linear, we can compute for $1 \leqq t \leqq m+1$ $\Delta^{M} g(t, v)\left(u_{1}, \cdots, u_{m+1}\right)$ obtaining (24).

$$
\Delta^{M} g(t, v)\left(u_{1}, \cdots, u_{m+1}\right)=\sum_{i=0}^{M} \Delta^{M} g_{k, m+1}(t, v)\left(u_{1}, \cdots, u_{m}\right) u_{m+1}^{k} \cdot
$$


Since a representation of form (21) holds for $t$ and the $M$ th difference annihilates all polynomials with degree less than $M, \Delta^{M} g(t, v)\left(u_{1}, \cdots\right.$, $\left.u_{m+1}\right)=0$. Thus, $\Delta^{w} g_{k, m+1}(t, v)\left(u_{1}, \cdots, u_{m}\right)=0$ for each $t$. By Lemma 5, $g_{k, m+1}\left(u_{1}, \cdots, u_{m}\right)$ is a polynomial in each $u_{t}$ separately. The induction hypothesis for $n=m$ ensures that $g_{k, m+1}\left(u_{1}, \cdots, u_{m}\right)$ is a polynomial in $u_{1}, \cdots, u_{m}$ together. By (22), $g\left(u_{1}, \cdots, u_{m+1}\right)$ is therefore a polynomial in $u_{1}, \cdots, u_{m+1}$. This establishes the induction step and completes the proof.

Lemma 10. A space $X$ is in $\mathscr{P}_{2 n}$ if and only if, for all $m$ and elements $x_{i},\left\|t_{0} x_{0}+\cdots+t_{m} x_{m}\right\|^{2 n}$ is a polynomial in $t_{0}, \cdots, t_{m}$.

Proof. It is easy to see that $\left\|t_{0} x_{0}+\cdots+t_{m} x_{m}\right\|^{2 n}$ must be a homogeneous polynomial if it is a polynomial at all, and that this condition is equivalent to $\left\|x_{0}+t_{1} x_{1}+\cdots+t_{m} x_{m}\right\|^{2 n}$ being a polynomial in $t_{1}, \cdots, t_{m}$.

If $\left\|x_{0}+t_{1} x_{1}+\cdots+t_{m} x_{m}\right\|^{2 n}$ is a polynomial, set $x_{0}=x, x_{1}=$ $y, x_{2}=\cdots=x_{m}=0$, arbitrary $x$ and $y$. We find that $X$ is in $\mathscr{P}_{2 n}$. To prove the converse, define $f\left(t_{1}, \cdots, t_{m}\right)=\left\|x_{0}+t_{1} x_{1}+\cdots+t_{m} x_{m}\right\|^{2 n}$. Fix all variables save $t_{r}$, then $f\left(t_{1}, \cdots, t_{m}\right)$ is a polynomial in $t_{r}$ with coefficients depending on $t_{1}, \cdots, \hat{t}_{r}, \cdots, t_{m}$. For any polynomial $p(t)=$ $\sum a_{k} t^{k}$ of degree $2 n$ we have the formulae $a_{k}=\sum_{j=0}^{2 n} c_{j, k} p(j)$, where $c_{j, k}$ can be found by solving the linear system: $\Sigma a_{k} j^{k}=p(j), 0 \leqq j \leqq$ $2 n$. Now $f\left(t_{1}, \cdots, t_{m}\right)$ can be put into form (25) for each $r$ and by the above, we can write $a_{k, r}\left(t_{1}, \cdots, \hat{t}_{r}, \cdots, t_{m}\right)=\sum_{j=0}^{2 n} c_{j, k} f\left(t_{1}, \cdots\right.$, $\left.j, \cdots, t_{m}\right)$.

$$
f\left(t_{1}, \cdots, t_{m}\right)=\sum_{k=0}^{2 n} a_{k, r}\left(t_{1}, \cdots, \hat{t}_{r}, \cdots, t_{m}\right) t_{r}^{k} .
$$

Hence $a_{k, r}\left(t_{1}, \cdots, \hat{t}_{r}, \cdots, t_{m}\right)$ is a sum of terms $\| x_{0}+t_{1} x_{1}+\cdots+j x_{r}+$ $t_{m} x_{m} \|^{2 n}$ each of which is continuous in $t_{1}, \cdots, \hat{t}_{r}, \cdots, t_{m}$. We can now apply Lemma 9 and conclude that $f\left(t_{1}, \cdots, t_{m}\right)$ is a polynomial in $t_{1}, \cdots, t_{m}$ jointly. As $\left|f\left(t_{1}, \cdots, t_{m}\right)\right| \leqq\left(\left\|x_{0}\right\|+\left|t_{1}\right|\left\|x_{1}\right\|+\cdots+\right.$ $\left.\left|t_{m}\right| \| x_{m}||\right)^{2 n}, f$ has degree at most $2 n$.

THEOREM 11. If an identity of the form (17) (with restrictions on constants as in Theorem 4) holds for one space in $\mathscr{P}_{2 n}$, then it holds for all spaces in $\mathscr{P}_{2 n}$. In particular, an identity holds in $\mathscr{P}_{2 n}$ if and only if it holds in Hilbert space.

Proof. An ordered $p$-tuple $\left(i_{0}, \cdots, i_{p-1}\right)$ of nonnegative integers is a $p$-partition of $d$ if $i_{0}+\cdots+i_{p-1}=d$. For each $p$ and $d$ there are a finite number of such partitions. Suppose (17) holds for $X$ in $\mathscr{P}_{2 n}$. The $i$ th $(m+1)$-partition of $t,(\pi(t, i, 0), \cdots, \pi(t, i, m))$ will be 
called $\pi(t, i)$ for short. We write $\pi(n, i)=\pi(i)$. In (17), we restrict $x_{i}$ to a one-dimensional subspace of $X$ generated by $x,\|x\|=1: x_{i}=$ $z_{i} x,\left\|x_{i}\right\|=\left|z_{i}\right|$, complex $z_{i}$. We obtain (26), which can be rewritten as (27) using $|z|^{2 n}=\left(|z|^{2}\right)^{n}$.

$$
\begin{gathered}
\sum_{k=0}^{r} \alpha_{k}\left|c_{k}(0) z_{0}+\cdots+c_{k}(m) z_{m}\right|^{2 n}=0 . \\
\sum_{k=0}^{r} a_{k}\left(\sum_{i=0}^{m} \sum_{j=0}^{m} c_{k}(i) \bar{c}_{k}(j) z_{i} \bar{z}_{j}\right)^{n}=0 .
\end{gathered}
$$

Index the $(m+1)$-partitions of $n$ from 1 to $s$, then (27) can be written as (28); where $c_{k}^{\pi_{p}}$ denotes $c_{k}(0)^{\pi_{p}(0)} \cdots c_{k}(m)^{\pi_{p}(m)}, z^{\pi_{p}}$ denotes $z_{0}^{\pi_{p}(0)} \cdots z_{m}^{\pi_{p}(m)}, \bar{c}_{k}^{\pi_{q}}$ and $\bar{z}^{\pi_{p}}$ are defined analogously, where the double sum ranges independently over all pairs of $(m+1)$-partitions of $n$ and where $d_{p, q}$ is the positive multinomial coefficient depending on $\pi_{p}$ and $\pi_{q}$.

$$
\sum_{k=0}^{r} \alpha_{k} \sum_{p=0}^{s} \sum_{q=0}^{s} d_{p, q} c_{k}^{\bar{c} p} \overline{\mathcal{C}}_{k}^{\pi} q \boldsymbol{z}^{\pi_{p}} \overline{\boldsymbol{z}}^{\pi_{q}}=0 .
$$

We now rewrite (28) as (29).

$$
\sum_{p=0}^{s} \sum_{q=0}^{s} d_{p, q}\left(\sum_{k=0}^{r} a_{k} c_{k}^{\bar{\pi}} \overline{\bar{c}_{k}} \bar{c}_{k}^{\pi}\right) z^{\pi} \bar{z}^{\pi_{q}}=0 .
$$

A polynomial in $z_{0}, \bar{z}_{0}, \cdots, z_{m}, \bar{z}_{m}$ which vanishes identically must have vanishing coefficients. As $d_{p, q}>0$ we deduce (30) for all partitions $\pi_{p}$ and $\pi_{q}$.

$$
\sum_{k=0}^{r} a_{k} c_{k}^{\pi} p \bar{c}_{k}^{\bar{\pi} q}=0
$$

Now let $Y$ be a space in $\mathscr{P}_{2 n}$ and fix elements $x_{0}, \cdots, x_{m}$ in $Y$. Write $f\left(z_{0}, \cdots, z_{m}\right)=\left\|z_{0} x_{0}+\cdots+z_{m} x_{m}\right\|^{2 n}$ for complex $z_{k}=v_{k}+i w_{k}$, $\left(v_{k}, w_{k}\right.$ real $)$. Then $f\left(z_{0}, \cdots, z_{m}\right)=\| v_{0} x_{0}+\cdots+v_{m} x_{m}+w_{0}\left(i x_{0}\right)+\cdots+$ $w_{m}\left(i x_{m}\right) \|^{2 n}$ is a polynomial of degree $2 n$ in $v_{0}, \cdots, v_{m}, w_{0}, \cdots, w_{m}$ by Lemma 10. From $2 v_{k}=z_{k}+\bar{z}_{k}, 2 i w_{k}=z_{k}-\bar{z}_{k}$, it follows that $f\left(z_{0}, \cdots, z_{m}\right)$ is a polynomial in $z_{k}$ and $\bar{z}_{k}$ of degree $2 n$. Rewrite $f\left(z_{0}, \cdots, z_{m}\right)$ in form (31), where the sum is taken over all $(m+1)$ partitions of $t$ and $2 n-t$, indexed by $j$ and $k$ respectively, where the $b_{t, j, k}$ 's are the coefficients and the condensed $z^{\pi(t, j)}$ is as before.

$$
f\left(z_{0}, \cdots, z_{m}\right)=\sum_{t=0}^{2 n} \sum_{\pi(t, j)} \sum_{\pi(2 n-t, k)} b_{t, j, k} z^{\pi(t, j)} \bar{z}^{\pi(2 n-t, k)} .
$$

Because $f\left(z_{0}, \cdots, z_{m}\right)=f\left(e^{i \theta} z_{0}, \cdots, e^{i \theta} z_{m}\right)$, (31) does not depend on $\theta$. Viewing (32) as a polynomial in $e^{i \theta}$ having as coefficients polynomials in $z$ and $\bar{z}$, it follows that $b_{t, j, k}=0$ unless $t=n$. 


$$
\sum_{t=0}^{n} e^{(2 t-2 n) i \theta} \sum_{\pi(t, j)} \sum_{\pi(2 n-t, j)} b_{t, j, k} z^{\pi(t, j)} \bar{z}^{\pi(2 n-t, k)} .
$$

We can thus rewrite (31) as (33), and insert this into (17).

$$
f\left(z_{0}, \cdots, z_{m}\right)=\sum_{p=0}^{s} \sum_{q=0}^{s} b_{p, q} z^{\pi_{p}} \bar{z}^{\pi_{q}} .
$$

We obtain (34).

$$
\begin{aligned}
& \sum_{k=0}^{r} a_{k}\left\|c_{k}(0) x_{0}+\cdots+c_{l k}(m) x_{m}\right\|^{2 n} \\
& =\sum_{k=0}^{r} a_{k}\left(\sum_{p=0}^{s} \sum_{q=0}^{s} b_{p, q} c_{k}^{\pi p} \overline{\mathcal{C}}_{k}^{\pi} q\right)=\sum_{p=0}^{s} \sum_{q=0}^{s} b_{p, q} \sum_{k=0}^{r} a_{k} c_{k}^{\pi} \bar{c}_{k}^{\bar{C}_{k} q}=0 .
\end{aligned}
$$

This last equality is a consequence of (30).

6. The parallelopiped law. Frechét [5] proved that any Banach space which satisfies (35) for all elements $x, y$, and $z$ is a Hilbert space.

$$
\begin{gathered}
\|x+y+z\|^{2}-\|x+y\|^{2}-\|x+z\|^{2}-\|y+z\|^{2} \\
+\|x\|^{2}+\|y\|^{2}+\|z\|^{2}=0 .
\end{gathered}
$$

Jordan and von Neumann reduced (35) to (1) by putting $z=-y$ and proving that the new condition is as strong as the old. The same sort of reduction applied to Theorem 12 will lead to a generalized parallelopiped law.

THEOREM 12. If $X$ is in $\mathscr{P}_{2 n}$ and $m>2 n$, then for all elements $x_{1}, \cdots, x_{m}$, equation (36) holds, where the inner sum is taken over all $k$-tuples $1 \leqq i_{1}<\cdots<i_{k} \leqq m$.

$$
\sum_{i=1}^{m}(-1)^{k} \sum\left\|x_{i_{1}}+\cdots+x_{i_{k}}\right\|^{2 n}=0 .
$$

Proof. In light of Theorem 11 it is sufficient to prove the theorem for elements in Hilbert space, indeed, we need only prove that (36) holds for all complex numbers $z_{j}$. The sum on the left-hand side is, in any case, a polynomial in the $z_{j}$ 's and $\bar{z}_{j}$ 's.

$$
\sum_{i=1}^{m}(-1)^{k} \Sigma\left|z_{i_{1}}+\cdots+z_{i_{k}}\right|^{2 n}=0 .
$$

A monomial $z_{j_{1}}^{r_{1}} \cdots z_{j_{s}}^{r_{s}} \cdot \bar{z}_{j_{1}^{s}}^{t_{s}} \cdots \bar{z}_{j_{s}}^{t_{s}}$ with $\Sigma \boldsymbol{r}_{k}=\Sigma t_{k}=n, r_{k}+t_{k}>0$ will occur in $\left|z_{i_{1}}+\cdots+z_{i_{k}}\right|^{2 n}$ either with multinomial coefficient

$$
(n !)^{2} /\left(r_{1} !\right) \cdots\left(r_{s} !\right)\left(t_{1} !\right) \cdots\left(t_{s} !\right)
$$

or not at all depending on whether the $j_{l}$ 's are contained in the $i_{k}$ 's or not. Because $r_{k}+t_{k}>0, s \leqq 2 n<m$, hence each monomial occurs 
in (37). Indeed, for $k \geqq s$, the $j_{l}$ 's are contained in precisely $\left(\begin{array}{c}m-s \\ k-s\end{array}\right)$ $k$-tuples and so, altogether, a monomial $z_{j_{1}}^{r_{1}} \cdots z_{j_{s}}^{r_{s}} \bar{z}_{z_{1}}^{t_{1}} \cdots \bar{z}_{j_{s}}^{t_{s}}$ will occur in (37) with total coefficient (38). As $\sum_{j=0}^{m-s}(-1)^{j}\left(\begin{array}{c}m-s \\ j\end{array}\right)=0$, the sum in (38) vanishes and so so (37) is proved.

$$
\sum_{k=s}^{m}(-1)^{k}\left(\begin{array}{r}
m-s \\
k-s
\end{array}\right) \frac{(n !)^{2}}{r_{1} ! \cdots r_{s} ! t_{1} ! \cdots t_{s} !} .
$$

The identity holds in Hilbert space and hence in all $\mathscr{P}_{2 n}$.

TheOREM 13 (The parallelopiped law). If $X$ is in $\mathscr{P}_{2 n}$ and $r>n$, then for all elements $x_{1}, \cdots, x_{r}$, the identity (39) holds in $X$, where the inner sum is taken over all choices of sign $\varepsilon_{i}= \pm 1$ and all ordered $k$-tuples with $1 \leqq i_{1}<\cdots<i_{k} \leqq r$.

$$
\sum_{k=1}^{r}(-1)^{k} 2^{r-k} \Sigma\left\|\varepsilon_{1} x_{i_{1}}+\cdots+\varepsilon_{k} x_{i_{k}}\right\|^{2 n}=0 .
$$

Proof. In Theorem 12, let $m=2 r, x_{2 k-1}=x_{k}^{\prime}$ and $x_{2 k}=-x_{k}^{\prime}$ for $1 \leqq k \leqq r$. Each term $\varepsilon_{1} x_{i_{1}}^{\prime}+\cdots+\varepsilon_{k} x_{i_{k}}^{\prime}$ will appear in (37) $2^{r-k}$ times depending on the joint inclusion or exclusion of the pairs $x_{2 j-1}+x_{2 j}$ for $j \neq i_{l}$. If the primes are dropped, (36) becomes (39) and the result follows.

Another identity which is satisfied in $\mathscr{P}_{2 n}$ is the following.

THEOREM 14. If $X$ is in $\mathscr{P}_{2 n}$ and $4 k>2 n$, then for all real $a_{i}$ with $\sum_{i=1}^{m} a_{i}=0$, equation (40) holds for all elements $x_{1}, \cdots, x_{m}$.

$$
\Sigma a_{i_{1}} \cdots a_{i_{2 k}}\left\|x_{i_{1}} \pm x_{i_{2}} \pm \cdots \pm x_{i_{2 k}}\right\|^{2 n}=0 \text {. }
$$

In (40), the inner sum is taken over all choices of sign \pm 1 as the $i_{j}$ 's range independently from 1 to $m$; the sum has $2^{2 k-1} m^{2 k}$ terms.

The proof is reserved for [11]. Krivine [9] introduced an inequality which determines whether a space $X$ is isometrically isomorphic to a subspace of $L_{p}$. For technical reasons, the proof in [9] fails when $p=2 n$. Theorem 14 illustrates what happens to Krivine's inequality in this case. Further implications will be considered in [11].

7. Koehler's $G_{2 n}$ spaces. In 1970, Koehler [8] defined a $G_{2 n}$ space to be a complex Banach space on which a form $\left\langle x_{1}, \cdots, x_{2 n}\right\rangle$ is defined satisfying (41)-(44).

$$
\langle x, \cdots, x\rangle=\|x\|^{2 n}
$$




$$
\begin{gathered}
\left\langle\overline{\left.x_{1}, \cdots, x_{2 n}\right\rangle}=\left\langle x_{2 n}, \cdots, x_{1}\right\rangle\right. \\
\langle\rangle \text { is linear in } x_{1}, \cdots, x_{n} \\
\langle\rangle \text { is conjugate linear in } x_{n+1}, \cdots, x_{2 n} \cdot
\end{gathered}
$$

Various properties of $G_{2 n}$ spaces are then discussed which parallel the development of Hilbert spaces. Among them are a proof that any $G_{2 n}$ space satisfies (39) and the construction through a polarization formula of a form $\left\langle x_{1}, \cdots, x_{2 n}\right\rangle$ on any complex Banach space which satisfies (39). Thus, by Theorem 6 , we can identify $G_{2 n}$ and $P_{2 n}$ and deduce the following corollary. (Note that one direction is immediate upon consideration of $\langle x+t y, \cdots, x+t y\rangle$.)

Corollary 15. A form $\left\langle x_{1}, \cdots, x_{2 n}\right\rangle$ satisfying (41)-(44) can be defined on a complex Banach space if and only if $\|x+t y\|^{2 n}$ is a polynomial in $t$ for every $x$ and $y$ in $X$.

8. Acknowledgments. The work contained herein constituted a portion of the author's doctoral dissertation at Stanford University written under the direction of Professor Per Enflo, to whom he expresses his gratitude. The author would also like to thank his undergraduate advisor, Professor W. A. J. Luxemburg, under whom he initiated his investigation of this problem.

\section{REFERENCES}

1. S. O. Carlsson, A characteristic property of Euclidean spaces, Arkiv Mat., 5 (1964), 327-330.

2. M. M. Day, Some characterizations of inner product spaces, Trans. Amer. Math. Soc., 62 (1947), 320-337.

3. - On criteria of Kasahara and Blumenthal for inner product spaces, Proc. of Amer. Math. Soc., 10 (1959), 92-100.

4. M. Fréchet, Toute Fonctionelle Continue est Développable en Série de Fonctionelles d'order Entier, C. R. Acad. Sci. (Paris), 148 (1909), 155-156.

5. — Sur la Définition Axiomatique d'une Classe d'espaces Vectoriel Distancés Applicables Vectoriellement sur l'espaces de Hilbert, Annals of Math., 36 (1935), 705-718.

6. G. G. Johnson, Inner products characterized by difference equations, Proc. Amer. Math. Soc., 37 (1973), 535-536.

7. P. Jordan and J. von Neumann, On inner products in linear metric spaces, Annals of Math., 36 (1935), 719-723.

8. D. O. Koehler, $G_{2 n}$ spaces, Trans. Amer. Math. Soc., 150 (1970), 507-518.

9. J-L. Krivine, Plongement des Espaces Normés Dans $L^{p}$ Pour $p>2$, C. R. Acad. Sci. (Paris), 261 (1965), 4307-4310.

10. E. R. Lorch, On certain implications which characterize Hilbert spaces, Annals of Math., 49 (1948), 523-532.

11. B. Reznick, Banach spaces with polynomial norms, Pacific J. Math., to appear.

12. D. A. Senechalle, A characterization of inner product spaces, Proc. Amer. Math. Soc., 19 (1968), 1306-1312. 
13. K. Sundaresan, Smooth Banach spaces, Math. Annalen, 173 (1967), 191-199.

14. W. H. Wilson, On a certain general class of functional equations, Amer. J. Math., 40 (1918), 263-282.

Received May 5, 1977 and in revised form July 27, 1977. The author was supported by the National Science Foundation.

DUKE UNIVERSITY

DURHAM, NC 27706 



\section{PACIFIC JOURNAL OF MATHEMATICS}

\section{EDITORS}

RICHARD ARENS (Managing Editor)

University of California

Los Angeles, California 90024

C. W. Curtis

University of Oregon

Eugene, OR 97403

C. C. MOORE

University of California

Berkeley, CA 94720

\section{J. DUGUNDJI}

Department of Mathematics University of Southern California Los Angeles, California 90007

R. Finn AND J. Milgram Stanford University Stanford, California 94305

ASSOCIATE EDITORS
E. F. BECKENBACH

B. H. NeUMANN

F. WOLF

K. YoshidA

\section{SUPPORTING INSTITUTIONS}

UNIVERSITY OF BRITISH COLUMBIA UNIVERSITY OF SOUTHERN CALIFORNIA CALIFORNIA INSTITUTE OF TECHNOLOGY STANFORD UNIVERSITY UNIVERSITY OF CALIFORNIA MONTANA STATE UNIVERSITY UNIVERSITY OF TOKYO UNIVERSITY OF NEVADA, RENO UNIVERSITY OF UTAH NEW MEXICO STATE UNIVERSITY OREGON STATE UNIVERSITY UNIVERSITY OF OREGON

WASHINGTON STATE UNIVERSITY UNIVERSITY OF WASHINGTON OSAKA UNIVERSITY 


\section{Pacific Journal of Mathematics}

\section{Vol. 74, No. $1 \quad$ May, 1978}

Gerald Arthur Anderson, Computation of the surgery obstruction groups

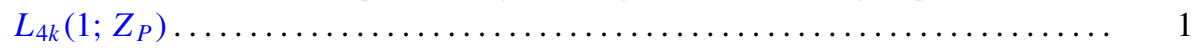

R. K. Beatson, The degree of monotone approximation ................ 5

Sterling K. Berberian, The character space of the algebra of regulated functions . . . 15

Douglas Michael Campbell and Jack Wayne Lamoreaux, Continua in the plane with

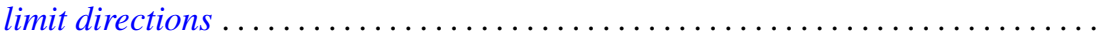

R. J. Duffin, Algorithms for localizing roots of a polynomial and the Pisot

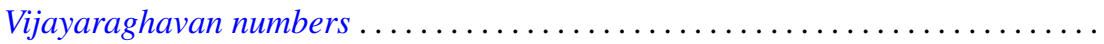

Alessandro Figà-Talamanca and Massimo A. Picardello, Functions that operate on

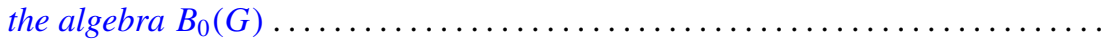

John Erik Fornaess, Biholomorphic mappings between weakly pseudoconvex

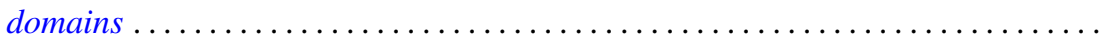

Andrzej Granas, Ronald Bernard Guenther and John Walter Lee, On a theorem of S.

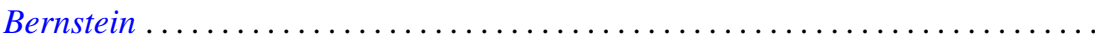

Jerry Grossman, On groups with specified lower central series quotients . .........

William H. Julian, Ray Mines, III and Fred Richman, Algebraic numbers, a constructive development . . . . . . . . . . . . . . . . . . . . . . .

Surjit Singh Khurana, A note on Radon-Nikodým theorem for finitely additive

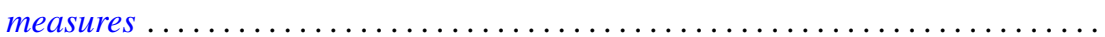

Garo K. Kiremidjian, A Nash-Moser-type implicit function theorem and nonlinear

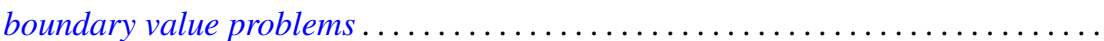

Ronald Jacob Leach, Coefficient estimates for certain multivalent functions ....

John Alan MacBain, Local and global bifurcation from normal eigenvalues. II . . 133

James A. MacDougall and Lowell G. Sweet, Three dimensional homogeneous algebras...

John Rowlay Martin, Fixed point sets of Peano continua ......

R. Daniel Mauldin, The boundedness of the Cantor-Bendixson order of some analytic sets...

Richard C. Metzler, Uniqueness of extensions of positive linear functions ..

Rodney V. Nillsen, Moment sequences obtained from restricted powers . .

Keiji Nishioka, Transcendental constants over the coefficient fields in differential elliptic function fields...

Gabriel Michael Miller Obi, An algebraic closed graph theorem

Richard Cranston Randell, Quotients of complete intersections by $\mathbf{C}^{*}$ actions . . 221

Bruce Reznick, Banach spaces which satisfy linear identities . .

Bennett Setzer, Elliptic curves over complex quadratic fields...

Arne Stray, A scheme for approximating bounded analytic functions on certain subsets of the unit disc.

Nicholas Th. Varopoulos, A remark on functions of bounded mean oscillation and bounded harmonic functions. Addendum to: "BMO functions and the

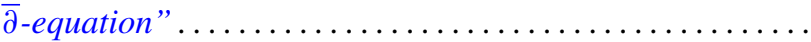

Charles Irvin Vinsonhaler, Torsion free abelian groups quasi-projective over their

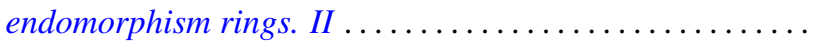

Thomas R. Wolf, Characters of $p^{\prime}$-degree in solvable groups ... 